\title{
Effects of Polyamines on Self-incompatibility-like Responses in Pollen Tubes of Citrus Cultivars, Banpeiyu and Hyuganatsu
}

\author{
Yiran Li and Asuka Uchida \\ Interdisciplinary Graduate School of Agriculture and Engineering, University of Miyazaki, 1-1, \\ Gakuenkibanadai-Nishi, Miyazaki 889-2192, Japan \\ Akiha Abe, Akihiro Yamamoto, Tomonari Hirano, and Hisato Kunitake'
Faculty of Agriculture, University of Miyazaki, 1-1, Gakuenkibanadai-Nishi, Miyazaki 889-2192,
Japan
}

Additional Index wORDs. HPLC, pollen culture, putrescine, spermidine, spermine, stylar crude protein

\begin{abstract}
Citrus is a genus with gametophytic self-incompatibility, which prevents fertilization by self-pollination. Polyamines (PAs), as a class of active small molecules, widely participate in various cellular activities. To investigate the effects of the changes in PA contents [putrescine, spermidine (SPD), and spermine] on pollen tube growth in self-incompatible (SI) plants, we cultured pollen of Citrus maxima 'Banpeiyu' and $C$. tamurana 'Hyuganatsu' in a Citrus mature pollen culture system and detected the PA content changes in pollen by high-performance liquid chromatography (HPLC) analysis during germination and after treatment with crude protein extracts of compatible or incompatible styles. We profiled the PA content changes during the germination course in pollen of 'Banpeiyu' and 'Hyuganatsu' by HPLC, and identified increases of free and perchloric-acid-insoluble SPD contents in pollen tubes in the incompatible treatment. Exogenous SPD treatment obviously increased the SPD contents in the pollen tube, inhibited pollen tube elongation, and resulted in some abnormal morphological alterations, such as tip inflation, tube content leakage and tip twist, which were observed in both 'Banpeiyu' and 'Hyuganatsu' pollen tubes exposed to incompatible treatment. This finding implied that SPD might participate in the SI response in Citrus.
\end{abstract}

Self-incompatibility is a genetic mechanism that exists in flowering plants to prevent a plant from being pollinated by its own pollen and to promote cross-pollination. Gametophytic SI (GSI), one form of SI, has been extensively studied and S-RNase models have been proposed to explain the mechanism of GSI in some species belonging to Rosaceae, Solanaceae, and Plantaginaceae (Hiratsuka et al., 2012; Lai et al., 2002; Lee et al., 1994; Murfett et al., 1994; Tao and Iezzoni, 2010). In these S-RNase models, $S$-allele are genes closely linked to the recognition of self-fertilization and the degradation of RNA in self-fertilized pollen tubes. Goldraij et al. (2006) proposed the compartmentalization model, which suggests that not only S-RNase but also other proteins, such as HT-B and $120 \mathrm{~K}$ proteins, participate in the recognition and degradation process. PrpS [Papaver rhoeas pollen $S$ (Wheeler et al., 2009)] and PrsS [P. rhoeas stigma $S$ (Foote et al., 1994)] were cloned from the pollen and stigma of Papaveraceae, respectively, as the first known self-incompatibility $(S)$ genes. A number of special molecules have been identified as participating in pollen-pistil interaction (Wheeler et al., 2009), but some details involved in these interactions are still far from well-known.

Polyamines are low-molecular-weight aliphatic polycations that exist in all living organisms (Cohen, 1998) and participate in various cellular regulations (Galston, 1983; Groppa and

Received for publication 14 Jan. 2015. Accepted for publication 23 Feb. 2015. We are grateful to Dr. Masahiro Mii of Graduate School of Horticulture, Chiba University, for his advice and critical reading of this manuscript. This study was supported by a Grant-in-Aid for Scientific Research (KAKENHI 25660029) from the Japan Society for the Promotion of Science (JSPS).

${ }^{1}$ Corresponding author. E-mail: hkuni@cc.miyazaki-u.ac.jp.
Benavides, 2008). The PAs, for example, putrescine (PUT), spermidine, and spermine (SPM), constitute a group of cell components that are precisely regulated. The biosynthesis and metabolism of PAs in plants have been well clarified. The oxidation of PAs can produce hydrogen peroxide $\left(\mathrm{H}_{2} \mathrm{O}_{2}\right)$, which has been shown to play important roles in cellular activities via signal transduction (Bagni and Tassoni, 2001; Hanfrey et al., 2001; Mattoo et al., 2010), and inhibition of the oxidation of spermidine (SPD), resulting in the germination failure of Arabidopsis thaliana pollen tube (Wu et al., 2010). The synthesis of PAs closely related to the maturation of Nicotiana tabacum pollen (Chibi et al., 1994) was shown to regulate the activity of RNase in the SI response of apple (Malus $\times$ domestica) pollen (Speranza et al., 1984). The germination rate of kiwifruit (Actinidia deliciosa) pollen was remarkably inhibited by methylglyoxal bis [guanylhydrazone (MGBG)] and by a high concentration of exogenous SPD (Antognoni and Bagni, 2008). In contrast, SPD added to a medium can reverse the inhibitory effect of D-arginine, a competitive inhibitor of arginine decarboxylase (ADC), on the germination rate and tube length of tomato pollen (Song et al., 2001). These findings suggest that PA contents in the pollen tube are precisely regulated, and that once the balance of PAs is disrupted, normal physiological growth and development are disturbed.

'Banpeiyu' and 'Hyuganatsu', belonging to the Rutaceae family, have strong GSI systems and produce only few seeds with self-pollination (Yamamoto et al., 2006). S-like RNase and CgF-box have been cloned from pummelo (Citrus grandis) cDNA library (Chai et al., 2011a, 2011b), while Miao et al. (2011) cloned the $S$-RNase homologous gene from mandarin 
cultivar Wuzishatangju (Citrus reticulata) shared 99\% amino acid sequence with $S$-like RNase of pummelo. Although some S-like RNases have been found to possess specific structures similar to that in Solanaceae, their exact function has not been identified. Although the S types of most Citrus species have been identified (Kim et al., 2011), the S-RNase and F-box have not yet been finally confirmed. Our previous works established a Citrus mature pollen culture system to provide a stable environment for pollen grain growth and succeeded in simulating SI-like responses in vitro using stylar crude protein extracts (Uchida et al., 2012). A high inhibition rate was observed after incompatible treatment accompanied with tip inflation, tube content leakage and tip twist. These morphological alterations largely coincided with the in vivo phenomena of Citrus pollen tubes arrested at the style whose walls developed irregular depositions of callose (Gentile et al., 2012), and the swollen tips of pollen tubes in incompatibly pollinated Pyrus pyrifolia (Hiratsuka et al., 1991) and Lilium hybrids (Niimi et al., 1997).

Unquestionably, PAs are necessary factors in Citrus pollen tube development, even though an accurate Citrus GSI model has not yet been identified. The aim of the present work was to explore the relationship between the PA content changes and SI in vitro as a way to clarify the SI mechanism in Citrus.

\section{Materials and Methods}

Plant materials. Mature anthers were collected just before anthesis in Spring 2013 from the flower buds of mature 'Banpeiyu' trees growing in the experimental field of University of Miyazaki and 'Hyuganatsu' trees growing in the experimental field of the Subtropical Plant Branch, Miyazaki Agricultural Research Institute. All anthers were separated from the buds and incubated at $25{ }^{\circ} \mathrm{C}$ overnight with a drying agent. Pollen grains collected from dehisced anthers were then stored at $-40{ }^{\circ} \mathrm{C}$. Styles from the pistils of 'Banpeiyu' and 'Hyuganatsu' were dissected, and immediately used for extracting stylar crude protein extracts.

PA CONTENT Changes during Germination. Dried pollen grains stored at $-40{ }^{\circ} \mathrm{C}$ were suspended in the Citrus mature pollen culture system $\left[0.04 \%\right.$ (w/v) $\mathrm{CaCl}_{2}, 0.01 \%$ (w/v) $\mathrm{H}_{3} \mathrm{BO}_{3}$, $0.0007 \%(\mathrm{w} / \mathrm{v}) \mathrm{KH}_{2} \mathrm{PO}_{4}, 10 \%(\mathrm{w} / \mathrm{v})$ sucrose, and $0.02 \%(\mathrm{w} / \mathrm{v})$ yeast extract, $\mathrm{pH}$ 7.0] as described previously (Uchida et al., 2012) at a density of $10^{6}$ grains $/ \mathrm{mL}$ for $0,2,4$, and $6 \mathrm{~h}$ cultivation at $25^{\circ} \mathrm{C}$ in the dark. Pollen tubes in the culture petri dish were collected in a clean $1.5-\mathrm{mL}$ tube, centrifuged at $20,000 \mathrm{~g}_{\mathrm{n}}$ for 10 min, and dried for weighing. The free, perchloric-acid (PCA)soluble, and PCA-insoluble PA contents in the germinated pollen were determined by high-performance liquid chromatography (LC-20; Shimadzu, Kyoto, Japan) after extraction with 0.5-M PCA (Scaramagli et al., 1995; Yamamoto et al., 2012). The PA contents were expressed on the basis of the dry weight of the pollen tubes. The experiments were repeated three times for statistical analysis via Tukey's multiple range test.

Preparation of STYlar CRUde protein EXTracts. Styles from 'Banpeiyu' and 'Hyuganatsu' were prepared for isolating the crude protein extracts using extraction buffer $[50 \mathrm{~mm}$ potassium phosphate buffer $\mathrm{pH} 7.0,10 \mathrm{~mm} \beta$-mercaptoethanol (Sigma Chemical Co., St. Louis, MO), 1\% (w/v) polyvinylpyrrolidone (Sigma Chemical Co.)]. Because the $\mathrm{S}$ protein in Citrus species has not been identified, we chose this extraction method to maintain the activities of proteins to a large extent. All the instruments were autoclaved before use. Two grams of styles were homogenized by mortar and pestle on an ice bath with 1-mL extraction buffer. The crude extracts homogenate was centrifuged at $14,000 \mathrm{~g}_{\mathrm{n}}$ for $10 \mathrm{~min}$ after a 30 -min ice bath with two to three times gently blending. The supernatant was divided into $100-\mu \mathrm{L}$ portions and stored in $200-\mu \mathrm{L}$ tubes at $-80{ }^{\circ} \mathrm{C}$ without loss of activity for about 10 months. The concentration of isolated protein was determined by the method of Bradford (Bradford, 1976) using Protein Assay Dye Reagent Concentrate (Bio-Rad Laboratories, Hercules, CA).

POLLEN GERMinAtion IN VITRO AND TREATMENT WITH STYLAR CRUde Protein EXtracts. Pollen grains of 'Banpeiyu' and 'Hyuganatsu' were resuspended and cultured in the Citrus mature pollen culture system and incubated for $4 \mathrm{~h}$ at $25^{\circ} \mathrm{C}$ in the dark. Then, stylar crude protein extracts of 'Banpeiyu' and 'Hyuganatsu' were individually added to the Citrus mature pollen culture medium of 'Banpeiyu' pollen tubes as the selfincompatible treatment (incompatible treatment) and crosscompatible treatment (compatible treatment), respectively. Similarly, we treated the 'Hyuganatsu' pollen with 'Banpeiyu' stylar crude protein extracts as the compatible treatment and the 'Hyuganatsu' extracts as the incompatible treatment. The working concentration of stylar crude protein extract was 50 $\mu \mathrm{g} \cdot \mathrm{mL}^{-1}$ (Uchida, 2012). After 2-h exposure to compatible and incompatible treatments, pollen tube growth was observed under a light microscope (BX51; Olympus, Tokyo, Japan), and the percentage of pollen grains with a pollen tube longer than the diameter of the pollen grain was calculated. Also, the inhibition rate of each treatment was assessed according to the shape of the tubes. We classified pollen tubes with twisted tips and content leakage as "inhibited pollen tubes." In total, 100 randomly chosen pollen grains per treatment were scored, and the experiment was repeated three times for statistical analysis via Tukey's multiple range test.

PA CONTENT ANALYSIS OF POLLEN TUBES WITH STYLAR CRUDE PROTEIN EXTRACTS AND EXOGENOUS SPD TREATMENTS IN VITRO. Pollen grains of 'Banpeiyu' and 'Hyuganatsu' were exposed to compatible and incompatible stylar crude protein extract treatments for $2 \mathrm{~h}$ by the methods described above. Extraction buffer alone was used as the control.

On the other hand, 4-h cultures of 'Banpeiyu' and 'Hyuganatsu' pollen tubes (initial culture) were exposed to exogenous SPD [free SPD (Sigma Chemical Co.)] at final concentrations of $0.1,0.5$, and $1.0 \mathrm{~mm}$, respectively, for $2 \mathrm{~h}$. Exogenous SPDtreated pollen tubes were photographed by light microscope (BX51). The inhibition rate was evaluated according to the evaluation method described above, and the lengths of the tubes were measured using Axio Vision (version 4.6; Carl Zeiss, Jena, Germany). One hundred randomly chosen pollen tubes per treatment were scored, and experiments on the inhibition rate and length measurement were repeated three times, respectively, for statistical analysis via Tukey's multiple range test.

Next, 'Banpeiyu' and 'Hyuganatsu' pollen tubes subjected to stylar crude protein extract treatment or 1.0-mM SPD treatment were collected in clean $1.5-\mathrm{mL}$ tubes for HPLC analysis as described above. The experiments were repeated three times for statistical analysis via Tukey's multiple range test.

\section{Results}

PA Content Changes during germination. We first analyzed the PA content changes during the development of pollen 
tubes in 'Banpeiyu' and 'Hyuganatsu' by HPLC analysis at different time points, including 0,2, 4, and $6 \mathrm{~h}$ in the Citrus mature pollen culture system without any addition.

In 'Banpeiyu', free and PCA-insoluble PUT (Fig. 1A), free and PCA-insoluble SPD (Fig. 1B), and free and PCA-soluble SPM (Fig. 1C) showed no significant changes after $6 \mathrm{~h}$ of cultivation. PCA-soluble PUT (Fig. 1A), PCA-soluble SPD (Fig. 1B), and PCA-insoluble SPM (Fig. 1C) showed slight increases. In 'Hyuganatsu', PCA-soluble and -insoluble PUT (Fig. 1D), free SPD (Fig. 1E), and PCA-soluble and -insoluble SPM (Fig. 1F) showed no significant changes after $6 \mathrm{~h}$ of cultivation. Free PUT showed a slight increase after $2 \mathrm{~h}$ of cultivation and remained at the same level for the following $4 \mathrm{~h}$
(Fig. 1D), while PCA-insoluble SPD (Fig. 1E) and free SPM (Fig. 1F) decreased after $6 \mathrm{~h}$ of cultivation.

Among the three types of PUT in 'Banpeiyu', the rate of free PUT was higher than that of PCA-insoluble PUT while the rate of PCA-soluble PUT was the lowest. The same trend was also observed in 'Hyuganatsu' (Fig. 1A and D). Although the PCAinsoluble SPD decreased in 'Hyuganatsu', no change occurred in 'Banpeiyu', and the content remained very high compared with that in the PCA-soluble and free types in both cultivars (Fig. 1B and E). During $6 \mathrm{~h}$ of cultivation, SPM levels of all three types were extremely low (Fig. 1C and F).

EFFECTS OF INCOMPATIBLE AND COMPATIBLE TREATMENTS ON PA Contents of POLlen tubes. Based on the previous results of

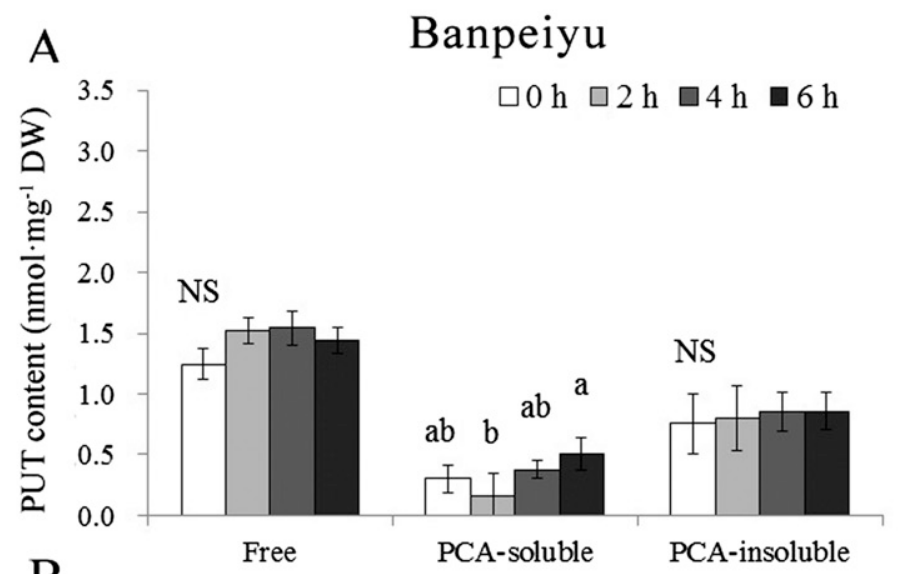

D Hyuganatsu
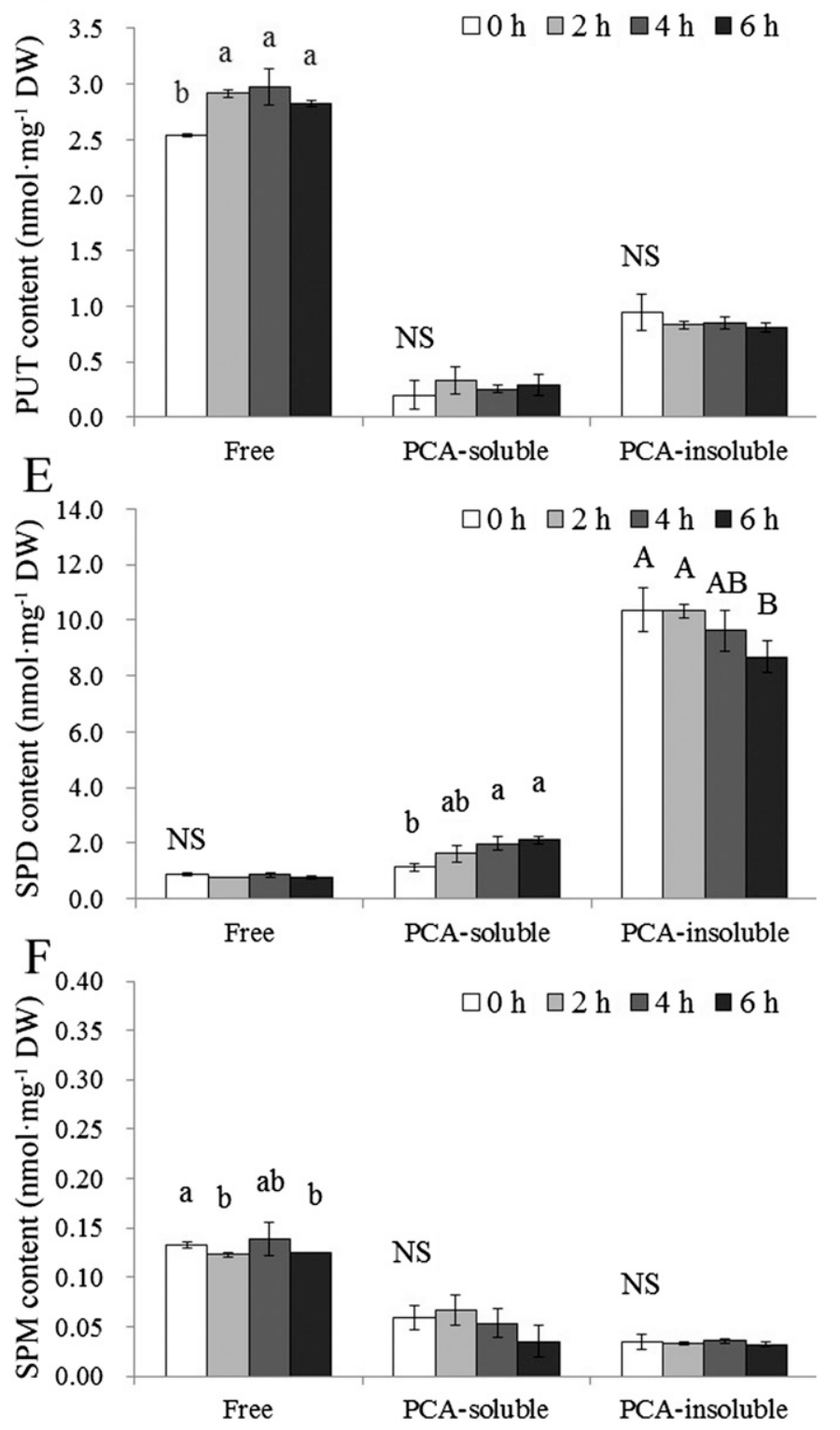

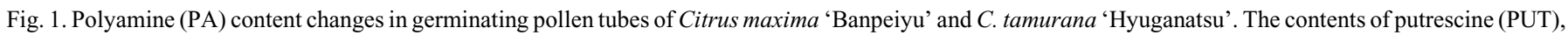
spermidine (SPD), and spermine (SPM) in 'Banpeiyu' (A-C) and 'Hyuganatsu' (D-F) pollen tubes were analyzed at the following time points: 0, 2, 4, and 6 h $(\mathrm{PCA}=$ perchloric-acid $)$. Each histogram shows mean $\pm \mathrm{SD}(n=3)$. Different letters represent significant differences at $5 \%$ level as determined by Tukey’s multiple range test (NS = no significant difference). 
PA content changes in the course of pollen grain germination, we added the stylar crude protein extracts after $4 \mathrm{~h}$ initial cultivation, to examine the changes in PA contents due to $2 \mathrm{~h}$ incompatible and compatible treatments, respectively. Pollen tubes exposed to incompatible treatment showed high inhibition rates compared with those exposed to compatible treatment and controls, and morphological alterations were confirmed (data not shown). We analyzed the PA contents of pollen tubes in 'Banpeiyu' and 'Hyuganatsu' by HPLC analysis.

In 'Banpeiyu', free PUT and SPM (Fig. 2A), PCA-soluble SPM (Fig. 2B), and PCA-insoluble PUT and SPM (Fig. 2C) showed no significant changes after the compatible or incompatible treatment compared with the control. The free SPD content was higher with the incompatible treatment than with the compatible treatment, while the latter was still higher than that of the control (Fig. 2A). The PCA-insoluble SPD content with the incompatible treatment was higher than that with the compatible treatment and that of the control, while the latter two did not differ from each other (Fig. 2C). However, both PCA-soluble PUT and SPD showed decreases with the compatible or incompatible treatment compared with the control (Fig. 2B). The three types of SPM remained at extremely low levels, showing no changes after the compatible or incompatible treatment.

In 'Hyuganatsu', the free and PCA-insoluble SPD contents showed increases after the incompatible treatment, but there was no significant difference between the compatible treatment and the control (Fig. 2D and F). As in the data for 'Banpeiyu,' the contents of all three types of SPM remained very low, showing no changes after the compatible and incompatible treatments compared with the control.

EFFECT OF EXOGENOUS SPD ON POLLEN TUBE GROWTH AND DEVELOPMENT. According to the results of PA content changes after compatible and incompatible treatments, the incompatible treatment induced increases in both free and PCA-insoluble SPD in the pollen tubes of both 'Banpeiyu' and 'Hyuganatsu'. A decrease in PCA-soluble SPD content with incompatible treatment was detected in 'Banpeiyu' but not in 'Hyuganatsu'. Among the PAs in the pollen tubes of 'Banpeiyu' and 'Hyuganatsu', the content of PCA-insoluble SPD was the highest (Fig. 1), and the change of its content was more noticeable than the others. Thus, we added SPD at three different concentrations $(0.1,0.5$, and $1.0 \mathrm{~mm})$ to the Citrus mature pollen culture system after $4 \mathrm{~h}$ initial cultivation. Pollen tube length was measured and pollen tubes were scored after exposure to SPD for $2 \mathrm{~h}$.

In 'Banpeiyu', the inhibition rates for the 0.5 - and $1.0-\mathrm{mm}$ SPD treatments were almost 15- and 22-fold higher than that of the control, respectively. However, the 0.1-mM exogenous SPD addition had little impact on the growth of the pollen tube (Fig. 3B). With SPD treatment at 0.5 and $1.0 \mathrm{~mm}$, the shape of the pollen tube showed the same changes as with the incompatible treatment, including tip twisting and tube content leakage (Fig. $3 \mathrm{~A}$, upper). At $1.0 \mathrm{~mm} \mathrm{SPD}$, pollen tube length was dramatically arrested in 'Banpeiyu' (Fig. 3C).

In 'Hyuganatsu', exogenous SPD also induced an increase in the inhibition rate, especially at concentrations of 0.5 and $1.0 \mathrm{~mm}$; in addition, the pollen tube shape showed varying degrees of tip twist and content leakage (Fig. 3A, lower). Pollen tube length measurement showed that SPD at 0.5 and $1.0 \mathrm{~mm}$ increases the inhibition rate (Fig. 3D) and arrests the growth of the pollen tube in 'Hyuganatsu' (Fig. 3E) as in 'Banpeiyu'.
Effect of exogenous SPD on PA contents of pollen TUBES. In 'Banpeiyu', the contents of free, PCA-soluble and PCA-insoluble SPD increased after exogenous application of SPD at $1.0 \mathrm{~mm}$ for $2 \mathrm{~h}$ compared with those of the control. However, the contents of the three types of PUT and SPM showed no significant differences from those of the control (Fig. 4A-C). The same results were obtained in 'Hyuganatsu' (Fig. 4D-F). These findings suggest that exogenous SPD only increased the PA contents of all three types of SPD, while the PUT and SPM were unaffected at all.

\section{Discussion}

In our study, an in vitro Citrus mature pollen culture system was used to reproduce the SI-like response in Citrus pollen tubes found in previous experiments done by our laboratory (Uchida, 2012). Thus, our in vitro culture system was found to be applicable for researches on role of PAs in the SI-like response.

Del Duca et al. (2010) first studied the relationship between SI and PAs in Pyrus communis with in vivo pollination. Gentile et al. (2012) investigated PA changes in the pummelo pistil after compatible and incompatible pollination in vivo. However, while those two previous studies demonstrated changes of total PAs including the pollen tube and pistil, they did not isolate the changes in the pollen tube. The Citrus mature pollen culture system supplied us a convenient approach to study the PA changes of the pollen tube independent of the pistil tissue.

PAs exist in plants as free, but also as soluble [conjugated with one organic acid, for example, cinnamic acid, coumaric acid or ferulic acid, or protein, easily dissolving in PCA or trichloroacetic acid (TCA)] and insoluble types (bi or trisubstituted formation and undissolved in PCA or TCA) (Bokern et al., 1995; Couée et al., 2004). In previous PAs analysis in apple (Bagni et al., 1981) and kiwifruit (Antognoni and Bagni, 2008; Falasca et al., 2010) pollen tubes contained different relative proportions of free, soluble and insoluble types during the germination course. From our results, we noticed that, in both 'Banpeiyu' and 'Hyuganatsu' pollen tubes, SPM of all three types was always at the very lowest concentrations (Fig. 1); this phenomenon was also observed in apple (Bagni et al., 1981) and kiwifruit (Antognoni and Bagni, 2008) pollen tubes throughout 2- and 4-h germination courses, respectively. PCAinsoluble SPD had the highest concentration in both 'Banpeiyu' and 'Hyuganatsu' pollen tubes (Fig. 1B and E), and it was also identified in the pollen germination course of kiwifruit (Antognoni and Bagni, 2008). However, the PA changes occurring in kiwifruit and apple pollen tubes during the course of germination were not completely identical, and we also noticed different trends for PA changes in 'Banpeiyu' and 'Hyuganatsu' pollen tubes, possibly due to the fruits being in different families.

We noticed that the level of PCA-insoluble SPD was always the highest of the PAs, with a slight decrease during pollen germination with a slight decrease (Fig. 1B and E), and it increased after incompatible treatment in both 'Banpeiyu' and 'Hyuganatsu' pollen tubes (Fig. 2C and F). Even though the results showed a sharp increase in the contents of free SPD, we thought the increased SPD was due to pollen tube absorption because we washed the tubes thoroughly to avoid any contamination by SPD residue. Meanwhile, increases of PCA-soluble 


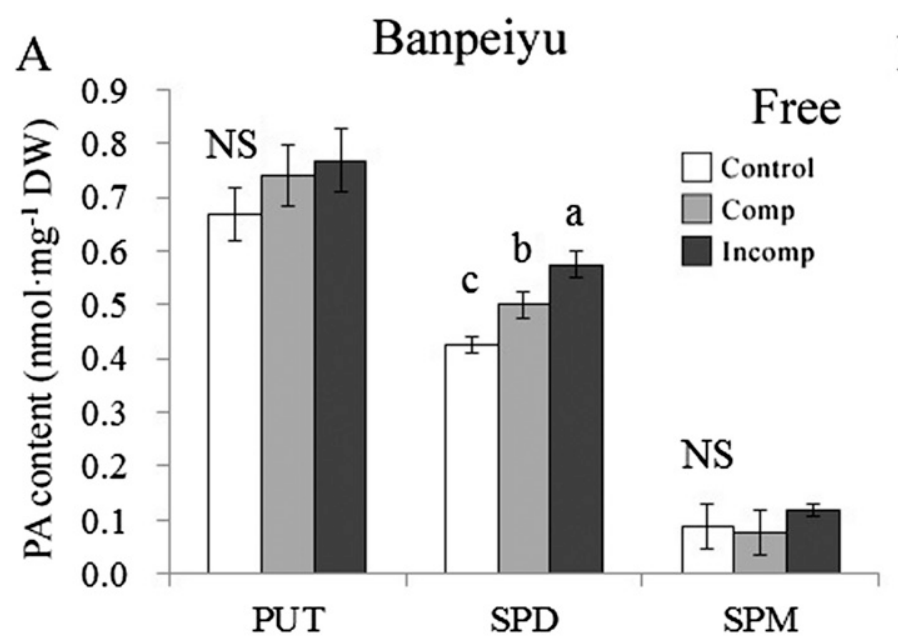

D Hyuganatsu
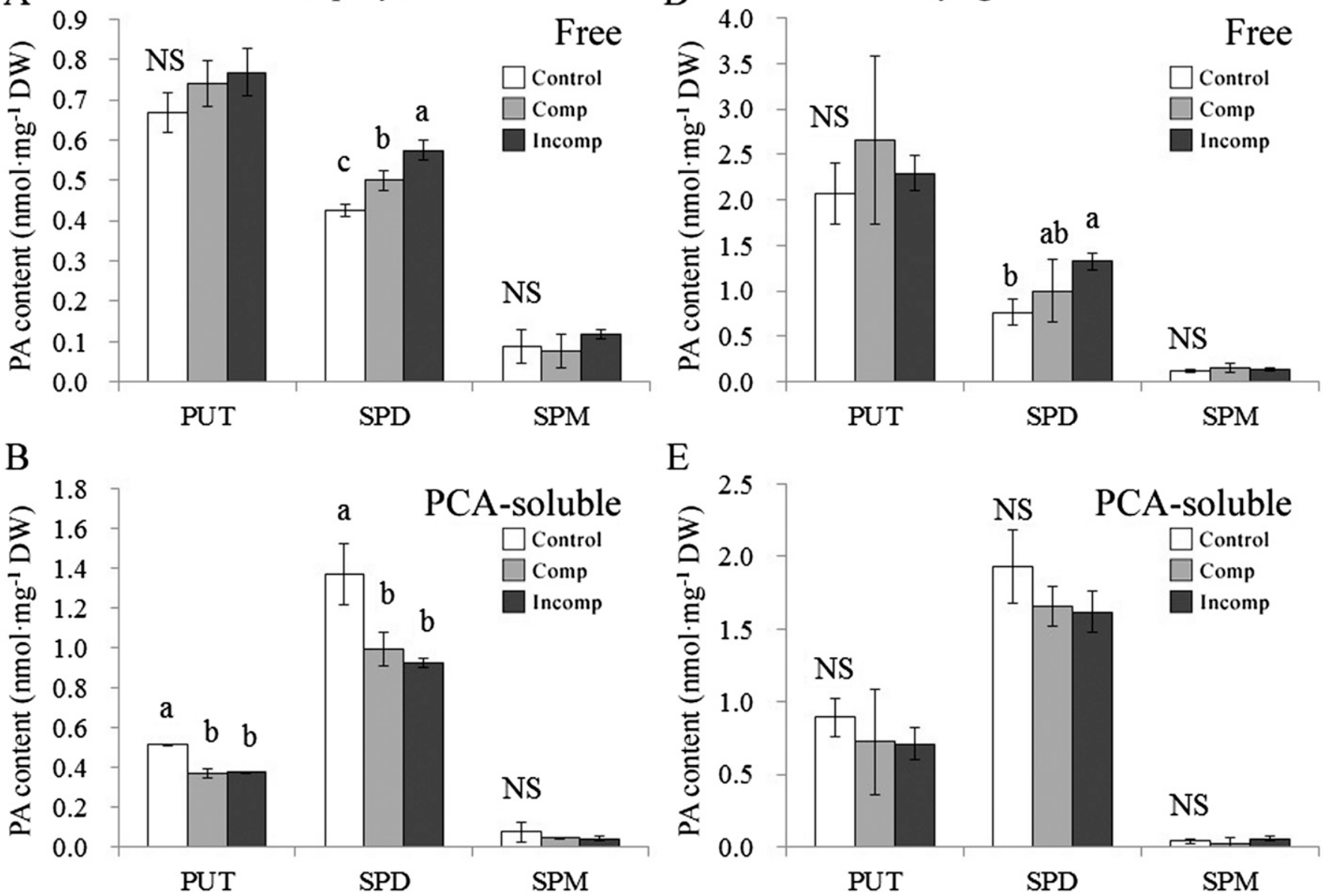

E
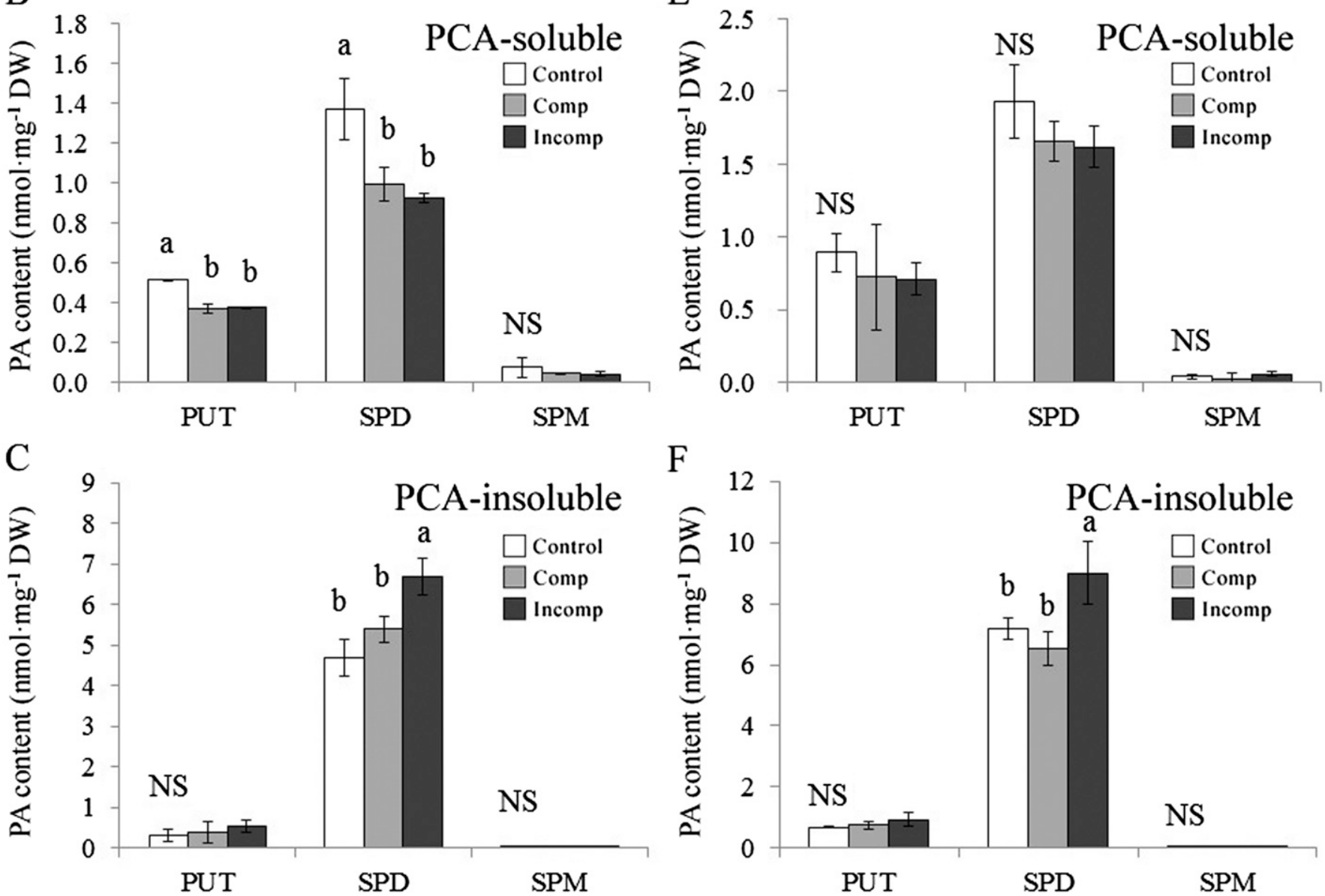

Fig. 2. Effects of compatible and incompatible treatments on PA contents in pollen tubes of Citrus maxima 'Banpeiyu' and C. tamurana 'Hyuganatsu'. Free, perchloric-acid (PCA)-soluble, and PCA-insoluble PA contents in pollen tubes with compatible (Comp) and incompatible (Incomp) treatments are shown for 'Banpeiyu' (A, B and C, respectively) and 'Hyuganatsu' (D, E, and F, respectively). Buffer was added into medium as a control (PUT $=$ putrescine, SPD $=$ spermidine, SPM = spermine). Each histogram shows mean $\pm \mathrm{SD}(n=3)$. Different letters represent significant differences at $5 \%$ level as determined by Tukey's multiple range test (NS = no significant difference).

and PCA-insoluble SPD were also detected; this suggested that the pollen tubes absorbed the exogenous SPD from the medium and transferred it into conjugated PA (Fig. 4). We also noticed that exogenous SPD certainly suppressed the tube length and altered its morphology (Fig. 3). Previous studies showed that the conjugated (PCA-soluble and/or -insoluble) PA particularly regulated the interaction with cell wall components (Lam et al., 1992) and the assembly of F-actin and microtubulin, in particular, in the pollen-tube growth process. Excessive PAs may saturate the actin-binding sites and hinder correct sitespecific assembly (Chowdhury et al., 1997). Therefore, maintaining the proper cytosolic PA contents in elongating pollen 


\section{Control}

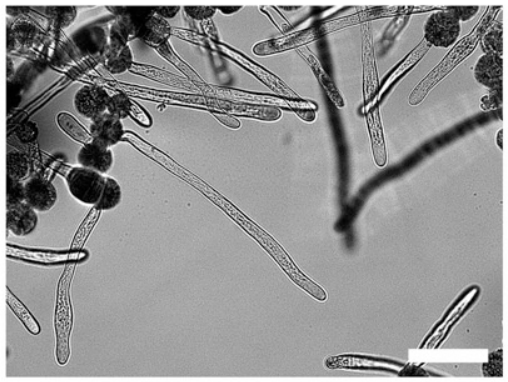

Banpeiyu

Hyuganatsu
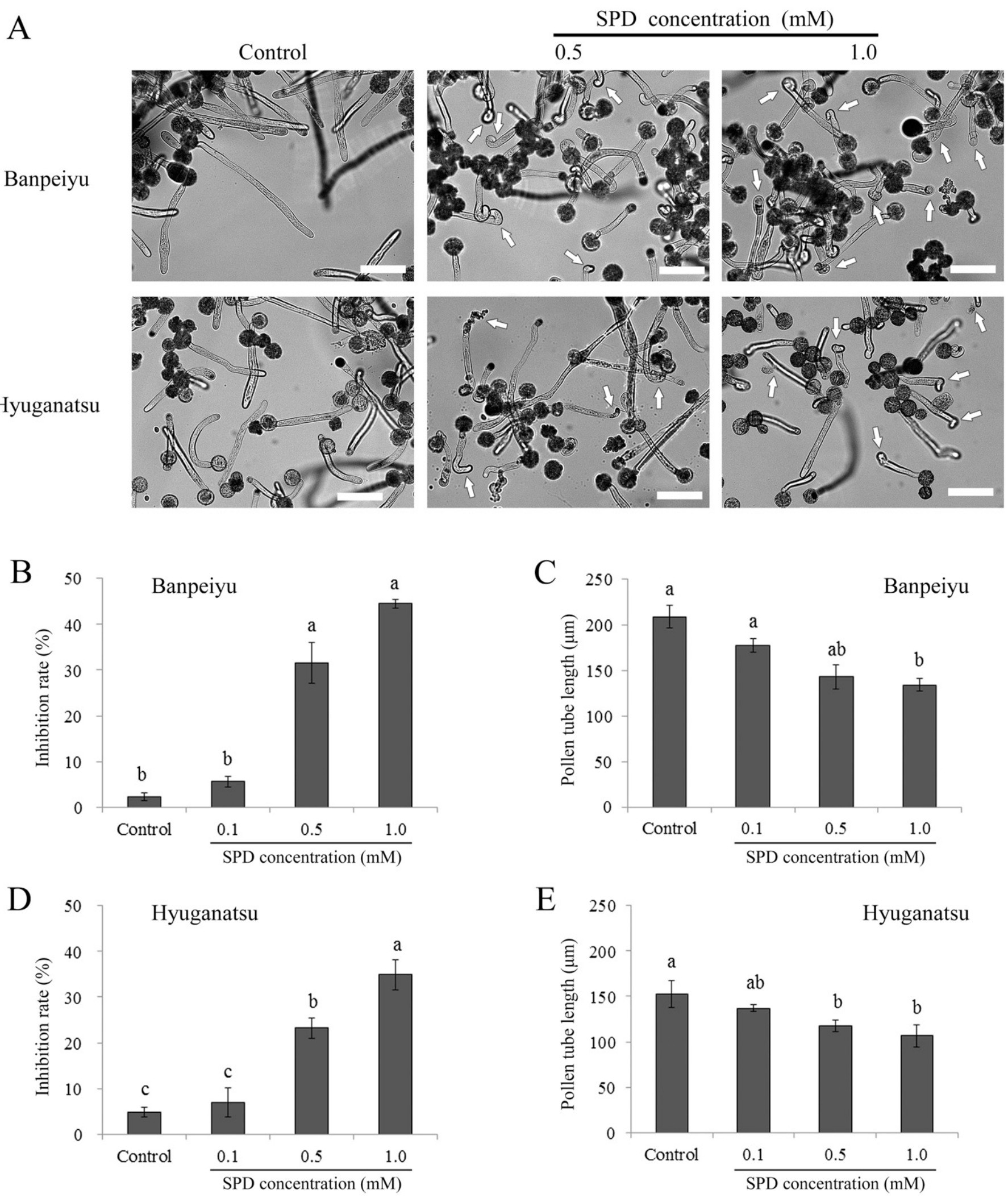

Fig. 3. Effect of exogenous SPD on pollen tube growth. (A) Pollen tube growth of Citrus maxima 'Banpeiyu' (upper) and C. tamurana 'Hyuganatsu' (lower) with 0.5 and $1.0 \mathrm{~mm}$ exogenous SPD addition. No SPD was added as a control. The white arrows indicate inhibited pollen tubes $(\mathrm{scale}$ bar $=100 \mu \mathrm{m})$. Effects of the concentration of exogenous SPD on the inhibition rate in pollen tubes of 'Banpeiyu' (B) and 'Hyuganatsu' (D). Effects of the concentration of exogenous SPD on the length of pollen tubes of 'Banpeiyu' $(\mathbf{C})$ and 'Hyuganatsu' $(\mathbf{E})$. One hundred randomly chosen pollen tubes per treatment were scored in experiments of inhibition rate and length measuring, respectively. Each histogram shows mean $\pm \mathrm{SD}(n=3)$. Different letters represent significant differences at $5 \%$ level as determined by Tukey's multiple range test. 

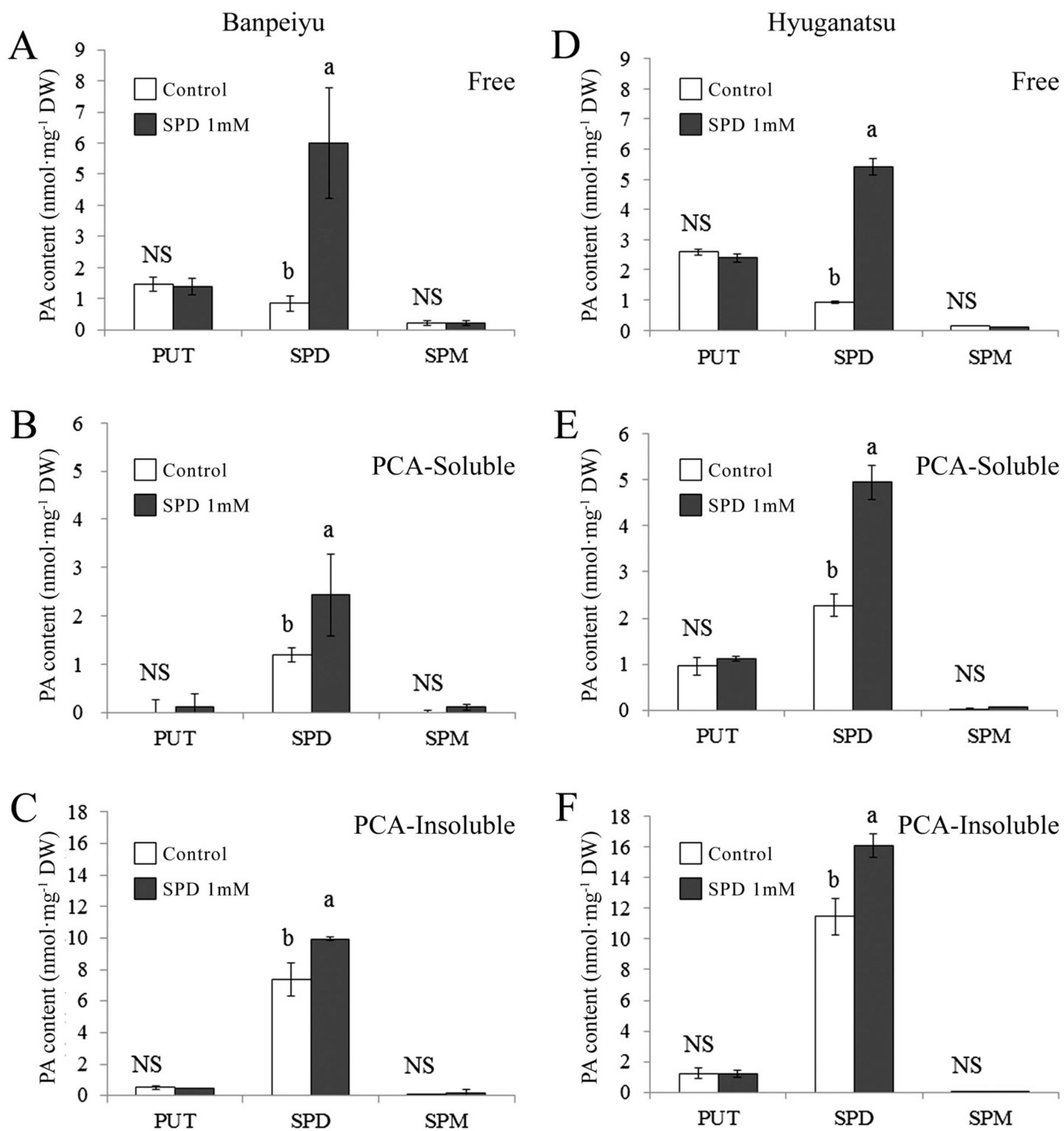

Fig. 4. Effect of exogenous SPD on the PUT, SPD, and SPM content of Citrus maxima 'Banpeiyu' and C. tamurana 'Hyuganatsu' pollen tubes. Free, perchloric-acid (PCA)-soluble, and PCA-insoluble PA content were compared between the treatments with 0 and $1 \mathrm{~mm}$ exogenous SPD addition in pollen tubes of 'Banpeiyu' (A, $\mathbf{B}$, and $\mathbf{C}$, respectively) and of 'Hyuganatsu' ( $\mathbf{D}, \mathbf{E}$, and $\mathbf{F}$, respectively). Each histogram shows mean $\pm \mathrm{SD}(n=3)$. Different letters represent significant differences at $5 \%$ level as determined by Tukey's multiple range test (NS = no significant difference).

tubes seems to be important, even if the relative proportions of free and conjugated PAs may vary among different plant species (Bagni and Tassoni, 2001).

In conclusion, we succeeded in profiling the proportions of PA contents in pollen grain germination of 'Banpeiyu' and 'Hyuganatsu', using a Citrus mature pollen culture system and
HPLC analysis. We identified an increase in SPD in pollen tubes subjected to incompatible treatment and exogenous SPD, and also observed the altered morphologies of the pollen tubes. These results implied that SPD might participate in the SI reaction of Citrus pollen tubes. Further research on cytological phenomena and related gene expression is required. 


\section{Literature Cited}

Antognoni, F. and N. Bagni. 2008. Bis(guanylhydrazones) negatively affect in vitro germination of kiwifruit pollen and alter the endogenous polyamine pool. Plant Biol. (Stuttg.) 10:334-341.

Bagni, N., P. Adamo, D. Serafini-Fracassini, and V.R. Villanueva. 1981. RNA, proteins and polyamines during tube growth in germinating apple pollen. Plant Physiol. 68:727-730.

Bagni, N. and A. Tassoni. 2001. Biosynthesis, oxidation and conjugation of aliphatic polyamines in higher plants. Amino Acids 20:301-317.

Bokern, M., L. Witte, V. Wray, M. Nimtz, and B. Meurer-Grimes. 1995. Trisubstituted hydroxycinnamic acid spermidines from Quercus dentata pollen. Phytochemistry 39:1371-1375.

Bradford, M.M. 1976. A rapid and sensitive method for the quantitation of microgram quantities of protein utilizing the principle of protein-dye binding. Anal. Biochem. 72:248-254.

Chai, L., X. Ge, M.K. Biswas, and X. Deng. 2011a. Molecular analysis and expression of a floral organ-relative F-box gene isolated from 'Zigui shatian' pummelo (Citrus grandis Osbeck). Mol. Biol. Rpt. 38:4429-4436.

Chai, L., X. Ge, Q. Xu, and X. Deng. 2011b. CgSL2, an S-like RNase gene in 'Zigui shatian' pummelo (Citrus grandis Osbeck), is involved in ovary senescence. Mol. Biol. Rpt. 38:1-8.

Chibi, F., A.J. Matilla, T. Angosto, D. Garrido, and C.H. Bornman. 1994. Changes in polyamine synthesis during anther development and pollen germination in tobacco (Nicotiana tabacum). Physiol. Plant. 92:61-68.

Chowdhury, Z.A., C. Barsigian, G.D. Chalupowicz, T.L. Bach, G. Garcia-Manero, and J. Martinez. 1997. Colocalization of tissue transglutaminase and stress fibers in human vascular smooth muscle cells and human umbilical vein endothelial cells. Expt. Cell Res. 231:38-49.

Cohen, S.S. 1998. Guide to the polyamines. Oxford Univ. Press, Oxford, UK.

Couée, I., I. Hummel, C. Sulmon, G. Gouesbet, and A. El Amrani. 2004. Involvement of polyamines in root development. Plant Cell Tissue Organ Cult. 76:1-10.

Del Duca, S., G. Cai, A. Di Sandro, and D. Serafini-Fracassini. 2010. Compatible and self-incompatible pollination in $P$. communis displays different polyamine levels and transglutaminase activity. Amino Acids 38:659-667.

Falasca, G., M. Franceschetti, N. Bagni, M.M. Altamura, and R. Biasi. 2010. Polyamine biosynthesis and control of the development of functional pollen in kiwifruit. Plant Physiol. Biochem. 48:565-573.

Foote, H.C., J.P. Ride, V.E. Franklin-Tong, E.A. Walker, M.J. Lawrence, and F.C. Franklin. 1994. Cloning and expression of a distinctive class of self-incompatibility $(S)$ gene from Papaver rhoeas L. Proc. Natl. Acad. Sci. USA 91:2265-2269.

Galston, A.W. 1983. Polyamines as modulators of plant development. Bioscience 33:382-388.

Gentile, A., F. Antognoni, R.A. Iorio, G. Distefano, G. Las Casas, S. La Malfa, D. Serafini-Fracassini, and S. del Duca. 2012. Polyamines and transglutaminase activity are involved in compatible and selfincompatible pollination of Citrus grandis. Amino Acids 42:1025-1035. Goldraij, A., K. Kondo, C.B. Lee, C.N. Hancock, M. Sivaguru, S. VazquezSantana, S. Kim, T.E. Phillips, F. Cruz-Garcia, and B. McClure. 2006. Compartmentalization of S-RNase and HT-B degradation in self-incompatible Nicotiana. Nature 439:805-810.

Groppa, M. and M. Benavides. 2008. Polyamines and abiotic stress: Recent advances. Amino Acids 34:35-45.

Hanfrey, C., S. Sommer, M.J. Mayer, D. Burtin, and A.J. Michael. 2001. Arabidopsis polyamine biosynthesis: Absence of ornithine decarboxylase and the mechanism of arginine decarboxylase activity. Plant J. 27:551-560.

Hiratsuka, S., M. Fujimura, T. Hayashida, Y. Nishikawa, and K. Nada. 2012. Pollen factors controlling self-incompatibility strength in Japanese pear. Sex. Plant Reprod. 25:347-352.
Hiratsuka, S., Y. Kitoh, and J. Matsushima. 1991. Induction of deformed pollen tube tips and their morphological characteristics in self-incompatible japanese pear (Pyrus pyrifolia). J. Jpn. Soc. Hort. Sci. 60:257-265.

Kim, J., T. Mori, and A. Wakana. 2011. Determination of selfincompatible Citrus cultivars with $S 1$ and/or $S 2$ alleles by pollination with homozygous $\mathrm{S}_{1}$ seedlings $\left(S_{1} S_{1}\right.$ or $\left.S_{2} S_{2}\right)$ of 'Banpeiyu' pummelo. J. Jpn. Soc. Hort. Sci. 80:404-413.

Lai, Z., W. Ma, B. Han, L. Liang, Y. Zhang, G. Hong, and Y. Xue. 2002. An F-box gene linked to the self-incompatibility $(S)$ locus of Antirrhinum is expressed specifically in pollen and tapetum. Plant Mol. Biol. 50:29-42.

Lam, T.B.T., K. Iiyama, and B.A. Stone. 1992. Cinnamic acid bridges between cell wall polymers in wheat and phalaris internodes. Phytochemistry 31:1179-1183.

Lee, H.S., S. Huang, and T. Kao. 1994. S proteins control rejection of incompatible pollen in Petunia inflata. Nature 367:560-563.

Mattoo, A.K., S.C. Minocha, R. Minocha, and A.K. Handa. 2010. Polyamines and cellular metabolism in plants: Transgenic approaches reveal different responses to diamine putrescine versus higher polyamines spermidine and spermine. Amino Acids 38:405413.

Miao, H.X., Y.H. Qin, J.A. Teixeira da Silva, Z.X. Ye, and G.B. Hu. 2011. Cloning and expression analysis of $S$-RNase homologous gene in Citrus reticulata Blanco cv. Wuzishatangju. Plant Sci. 180:358367 .

Murfett, J., T.L. Atherton, B. Mou, C.S. Gasser, and B.A. McClure. 1994. S-RNase expressed in transgenic Nicotiana causes S-allelespecific pollen rejection. Nature 367:563-566.

Niimi, Y., T.H. Li, and K. Matsuo. 1997. Self-compatible andincompatible reactions in asiatic hybrid Lilium $\times$ 'Enchantment': Influence of pisti age on seed set. J. Jpn. Soc. Hort. Sci. 65:835-842.

Scaramagli, S., M. Bueno, P. Torrigiani, M. Maddalena Altamura, F. Capitani, and N. Bagni. 1995. Morphogenesis in cultured thin layers and pith explants of tobacco. II. Early hormone-modulated polyamine biosynthesis. J. Plant Physiol. 147:113-117.

Song, J., K. Nada, and S. Tachibana. 2001. The early increase of S-adenosylmethionine decarboxylase activity is essential for the normal germination and tube growth in tomato (Lycopersicon esculentum Mill.) pollen. Plant Sci. 161:507-515.

Speranza, A., G. Calzoni, and N. Bagni. 1984. Evidence for a polyamine-mediated control of ribonuclease activity in germinating apple pollen. Physiol. Veg. 22:323-331.

Tao, R. and A.F. Iezzoni. 2010. The S-RNase-based gametophytic self-incompatibility system in Prunus exhibits distinct genetic and molecular features. Sci. Hort. 124:423-433.

Uchida, A. 2012. Fundamental studies on self-incompatibility in Citrus species. Ph.D. Thesis, Univ. Miyazaki, Miyazaki City, Japan.

Uchida, A., A. Abe, Y. Hoshino, and H. Kunitake. 2012. Liquid culture system for mature pollen in Hyuganatsu (Citrus tamurana hort. ex Tanaka). Hort. Res. (Japan) 11:173-179.

Wheeler, M.J., B.H. de Graaf, N. Hadjiosif, R.M. Perry, N.S. Poulter, K. Osman, S. Vatovec, A. Harper, F.C. Franklin, and V.E. FranklinTong. 2009. Identification of the pollen self-incompatibility determinant in Papaver rhoeas. Nature 459:992-995.

Wu, J., Z. Shang, J. Wu, X. Jiang, P.N. Moschou, W. Sun, K.A. Roubelakis-Angelakis, and S. Zhang. 2010. Spermidine oxidasederived $\mathrm{H}_{2} \mathrm{O}_{2}$ regulates pollen plasma membrane hyperpolarizationactivated $\mathrm{Ca}^{2+}$-permeable channels and pollen tube growth. Plant $\mathrm{J}$. 63:1042-1053.

Yamamoto, A., I.-S. Shim, and S. Fujihara. 2012. Chilling-stress responses by rice seedlings grown with different ammonium concentrations and its relationship to leaf spermidine content. J. Plant Biol. 55:191-197.

Yamamoto, M., T. Kubo, and S. Tominaga. 2006. Self-and crossincompatibility of various Citrus accessions. J. Jpn. Soc. Hort. Sci. 75:372-378. 